\title{
Quantization Dimension Function and Ergodic Measure with Bounded Distortion
}

by

\author{
Mrinal Kanti ROYCHOWDHURY
}

Presented by Czesław RYLL-NARDZEWSKI

Summary. The quantization dimension function for the image measure of a shift-invariant ergodic measure with bounded distortion on a self-conformal set is determined, and its relationship to the temperature function of the thermodynamic formalism arising in multifractal analysis is established.

1. Introduction. The term quantization in this paper refers to the idea of estimating a given probability on $\mathbb{R}^{d}$ with a discrete probability, that is, a "quantized" version of the probability supported on a finite set. Following the work of Graf and Luschgy (cf. GL1, GL2]), we define the quantization dimension (or perhaps better, the quantization dimension function) as follows. Given a Borel probability measure $\mu$ on $\mathbb{R}^{d}$, a number $r \in(0,+\infty)$ and a natural number $n \in \mathbb{N}$, the $n$th quantization error of order $r$ for $\mu$ is defined by

$$
e_{n, r}=\inf \left\{\left(\int d(x, \alpha)^{r} d \mu(x)\right)^{1 / r}: \alpha \subset \mathbb{R}^{d}, \operatorname{card}(\alpha) \leq n\right\}
$$

where $d(x, \alpha)$ denotes the distance from the point $x$ to the set $\alpha$ with respect to a given norm $\|\cdot\|$ on $\mathbb{R}^{d}$. We note that if $\int\|x\|^{r} d \mu(x)<\infty$ then there is some set $\alpha$ for which the infimum is achieved (cf. GL1]). The quantization dimension of order $r$ for $\mu$ is defined to be

$$
D_{r}=D_{r}(\mu)=\lim _{n \rightarrow \infty} \frac{\log n}{-\log e_{n, r}},
$$

2010 Mathematics Subject Classification: Primary 37A50; Secondary 28A80, 94A34.

Key words and phrases: ergodic measure with bounded distortion, conformal iterated function system, quantization dimension, temperature function. 
if the limit exists. If the limit does not exist then we define $\bar{D}_{r}$ as the lim sup of the sequence and $\underline{D}_{r}$ as the liminf. Graf and Luschgy also define $e_{n, r}$ for $r=0$ and $r=+\infty$, but in this paper we only deal with the case $0<r<+\infty$. One sees that the quantization dimension is actually a function $r \mapsto D_{r}$ which measures the asymptotic rate at which $e_{n, r}$ goes to zero. If $D_{r}$ exists, then one can write

$$
\log e_{n, r} \sim \log (1 / n)^{1 / D_{r}} .
$$

Graf and Luschgy determined a formula for the quantization dimension function for a self-similar probability measure $\mu$ defined for an iterated function system using a finite number of contracting similarity mappings $\phi_{1}, \ldots, \phi_{N}$ on $\mathbb{R}^{d}$ satisfying the open set condition, and given a probability vector $\left(p_{1}, \ldots, p_{N}\right)$. The measure $\mu$ satisfies

$$
\mu=\sum_{i=1}^{N} p_{i} \mu \circ \phi_{i}^{-1} .
$$

They showed that $D_{r}:=D_{r}(\mu)$ satisfies

$$
\sum_{i=1}^{N}\left(p_{i} s_{i}^{r}\right)^{D_{r} /\left(r+D_{r}\right)}=1,
$$

where $s_{i}$ is the contraction coefficient for the mapping $\phi_{i}$. Note that from (1) it is clear that the quantization dimension for a self-similar probability measure has a relationship to the temperature function arising in the thermodynamic formalism of multifractal analysis (cf. [F1]). The above result was extended by Lindsay and Mauldin to $F$-conformal measures with finitely many conformal mappings (cf. $[\mathrm{LM}]$ ). In $[\mathrm{R}]$, we determined the quantization dimension function for the image measure of a Gibbs measure induced on the coding space via the coding map on a self-similar set, and showed its functional relationship to the temperature function of the thermodynamic formalism. In this paper, the quantization dimension function for the image measure of a shift-invariant ergodic measure with bounded distortion on a self-conformal set is determined, and its relationship to the temperature function of the thermodynamic formalism arising in multifractal analysis is established.

2. Basic definitions, lemmas and propositions. Let us write

$$
\begin{aligned}
& V_{n, r}=\inf \left\{\int d(x, \alpha)^{r} d \mu(x): \alpha \subset \mathbb{R}^{d}, \operatorname{card}(\alpha) \leq n\right\}, \\
& u_{n, r}=\inf \left\{\int d\left(x, \alpha \cup U^{c}\right)^{r} d \mu(x): \alpha \subset \mathbb{R}^{d}, \operatorname{card}(\alpha) \leq n\right\},
\end{aligned}
$$


where $U$ is a set which comes from the open set condition (definition follows) and $U^{c}$ denotes the complement of $U$. We see that

$$
u_{n, r}^{1 / r} \leq V_{n, r}^{1 / r}=e_{n, r} .
$$

We will call sets $\alpha_{n} \subset \mathbb{R}^{d}$ for which the above infimums are achieved $n$-optimal sets for $e_{n, r}, V_{n, r}$ or $u_{n, r}$ respectively. As stated above, Graf and Luschgy have shown that $n$-optimal sets exist when $\int\|x\|^{r} d \mu(x)<\infty$.

Let $V \subset \mathbb{R}^{d}$ be an open set. A $\mathcal{C}^{1}$-map $\phi: V \rightarrow \mathbb{R}^{d}$ is conformal if the differential $\phi^{\prime}(x): \mathbb{R}^{d} \rightarrow \mathbb{R}^{d}$ satisfies $\left|\phi^{\prime}(x) y\right|=\left|\phi^{\prime}(x)\right| \cdot|y| \neq 0$ for all $x \in V$ and $y \in \mathbb{R}^{d}, y \neq 0 ;\left|\phi^{\prime}(x)\right|$ represents the norm of the derivative at $x \in \mathbb{R}^{d}$. Furthermore, $\phi: V \rightarrow \mathbb{R}^{d}$ is contracting if there exists $0<\gamma<1$ such that $|\phi(x)-\phi(y)| \leq \gamma|x-y|$ for all $x, y \in V$. We say that $\left\{\phi_{i}: X \rightarrow X\right\}_{i=1}^{N}$ is a conformal iterated function system (conformal IFS) on a compact set $X \subset \mathbb{R}^{d}$ if each $\phi_{i}$ extends to an injective contracting conformal map $\phi_{i}: V \rightarrow V$ on an open set $V \supset X$.

Let $\left\{\phi_{i}\right\}_{i=1}^{N}$ be a conformal IFS on a compact set $X \subset \mathbb{R}^{d}$ for some finite $N \geq 2$ such that $\left\|\phi_{i}^{\prime}\right\| \leq s<1$ for some $s$ where $\left\|\phi_{i}^{\prime}\right\|$ denotes the supremum norm of the derivative.

Let $\Sigma=\{1, \ldots, N\}^{\mathbb{N}}$ be the code space over the indices $1, \ldots, N$. Let $\Sigma_{n}=\{1, \ldots, N\}^{n}$, and $\Sigma_{*}=\bigcup_{n=0}^{\infty} \Sigma_{n}$ be the set of all sequences of finite length (also called words) including the empty sequence $\emptyset$. For $\omega=$ $\left(\omega_{1}, \ldots, \omega_{n}\right) \in \Sigma_{n}$ we write $|\omega|=n$ to denote the length $n$ of $\omega$, and $\left.\omega\right|_{k}=\left(\omega_{1}, \ldots, \omega_{k}\right), k \leq n$, to denote the truncation of $\omega$ to the length $k$. We write $\omega \tau=\omega * \tau=\left(\omega_{1}, \ldots, \omega_{|\omega|}, \tau_{1}, \tau_{2}, \ldots\right)$ for the juxtaposition of $\omega=\left(\omega_{1}, \ldots, \omega_{|\omega|}\right) \in \Sigma_{*}$ and $\tau=\left(\tau_{1}, \tau_{2}, \ldots\right) \in \Sigma_{*} \cup \Sigma$. For $\omega \in \Sigma_{*}$ and $\tau \in \Sigma_{*} \cup \Sigma$ we say $\tau$ is an extension of $\omega$, written as $\omega \prec \tau$, if $\left.\tau\right|_{|\omega|}=\omega$. For $\omega=\left(\omega_{1}, \ldots, \omega_{|\omega|}\right) \in \Sigma_{*}$ we set

$$
\begin{aligned}
& \omega^{-}= \begin{cases}\emptyset, & |\omega|=1, \\
\left(\omega_{1}, \ldots, \omega_{|\omega|-1}\right), & |\omega|>1,\end{cases} \\
& \phi_{\omega}= \begin{cases}\operatorname{Id}_{\mathbb{R}^{d}}, & \omega=\emptyset, \\
\phi_{\omega_{1}} \circ \cdots \circ \phi_{\omega_{|\omega|}}, & |\omega| \geq 1 .\end{cases}
\end{aligned}
$$

Let $\hat{\mu}$ be a shift-invariant ergodic measure on $\Sigma$ satisfying the bounded distortion property, i.e., there exists a constant $K \geq 1$ such that

$$
K^{-1} \hat{\mu}([\omega]) \hat{\mu}([\tau]) \leq \hat{\mu}([\omega \tau]) \leq K \hat{\mu}([\omega]) \hat{\mu}([\tau])
$$

for any two words $\omega$ and $\tau$ in $\Sigma_{*}$. Since given $\omega=\left(\omega_{i}\right)_{i=1}^{\infty} \in \Sigma$, the diameters of the compact sets $\phi_{\omega \mid n}(X)=\phi_{\omega_{1}} \circ \cdots \circ \phi_{\omega_{n}}(X), n \geq 1$, converge to zero and since they form a descending family, the set

$$
\bigcap_{n=0}^{\infty} \phi_{\omega \mid n}(X)
$$


is a singleton and therefore, if we denote its element by $\pi(\omega)$, this defines the coding map $\pi: \Sigma \rightarrow X$. The main object of our interest is the limit set

$$
J=\pi(\Sigma)=\bigcup_{\omega \in \Sigma} \bigcap_{n=1}^{\infty} \phi_{\omega \mid n}(X) .
$$

Note that $J$ satisfies the natural invariance equality $J=\bigcup_{i=1}^{N} \phi_{i}(J)$, and is called the self-conformal set corresponding to the conformal IFS. Let us assume that the iterated function system satisfies the open set condition, i.e., there exists a non-empty open set $U \subset X$ such that $\phi_{i}(U) \subset U$ for every $1 \leq i \leq N$ and $\phi_{i}(U) \cap \phi_{j}(U) \neq \emptyset$ for every pair $i, j$ in $\{1, \ldots, N\}$ with $i \neq j$. Furthermore, the system satisfies the strong open set condition if $U$ can be chosen such that $U \cap J \neq \emptyset$. Note that in the case of a conformal iterated function system using a finite number of mappings, the open set condition implies the strong open set condition (cf. [P-S] $)$. Hence, in our case if $U$ is the open set from the open set condition, then it also satisfies $U \cap J \neq \emptyset$.

The following two lemmas for conformal iterated function systems are borrowed from Patzschke (cf. [P]).

Lemma 2.1. There exists a constant $C \geq 1$ such that $\left|\phi_{\omega}^{\prime}(y)\right| \leq C\left|\phi_{\omega}^{\prime}(x)\right|$ for every $\omega \in \Sigma_{*}$ and every pair of points $x, y \in V$.

LEMMA 2.2. There exists a constant $\tilde{C} \geq C$ such that

$$
\tilde{C}^{-1}\left\|\phi_{\omega}^{\prime}\right\| d(x, y) \leq d\left(\phi_{\omega}(x), \phi_{\omega}(y)\right) \leq \tilde{C}\left\|\phi_{\omega}^{\prime}\right\| d(x, y)
$$

for every $\omega \in \Sigma_{*}$ and every pair of points $x, y \in V$, where $d$ is the metric on $X$.

The following lemma plays a vital role in this paper.

Lemma 2.3. For any $\omega, \tau \in \Sigma_{*}$ and any $t \in \mathbb{R}$,

$$
C^{-(t)}\left\|\phi_{\omega}^{\prime}\right\|^{t}\left\|\phi_{\tau}^{\prime}\right\|^{t} \leq\left\|\phi_{\omega \tau}^{\prime}\right\|^{t} \leq C^{(t)}\left\|\phi_{\omega}^{\prime}\right\|^{t}\left\|\phi_{\tau}^{\prime}\right\|^{t},
$$

where

$$
C^{(t)}= \begin{cases}C^{t} & \text { if } t \geq 0 \\ C^{-t} & \text { if } t<0\end{cases}
$$

and $C^{-(t)}=\left(C^{(t)}\right)^{-1}$.

Proof. For any $\omega, \tau \in \Sigma_{*}$ and any $x \in X$ with $y=\phi_{\tau}(x)$ we know that $\phi_{\omega \tau}^{\prime}(x)=\phi_{\omega}^{\prime}(y) \phi_{\tau}^{\prime}(x)$. Hence by Lemma 2.1 we have

$$
C^{-1}\left|\phi_{\omega}^{\prime}(x)\right|\left|\phi_{\tau}^{\prime}(x)\right| \leq\left|\phi_{\omega \tau}^{\prime}(x)\right| \leq C\left|\phi_{\omega}^{\prime}(x)\right|\left|\phi_{\tau}^{\prime}(x)\right|
$$

and thus $C^{-1}\left\|\phi_{\omega}^{\prime}\right\|\left\|\phi_{\tau}^{\prime}\right\| \leq\left\|\phi_{\omega \tau}^{\prime}\right\| \leq C\left\|\phi_{\omega}^{\prime}\right\|\left\|\phi_{\tau}^{\prime}\right\|$. Thus for any $t \geq 0$ we have

$$
C^{-t}\left\|\phi_{\omega}^{\prime}\right\|^{t}\left\|\phi_{\tau}^{\prime}\right\|^{t} \leq\left\|\phi_{\omega \tau}^{\prime}\right\|^{t} \leq C^{t}\left\|\phi_{\omega}^{\prime}\right\|^{t}\left\|\phi_{\tau}^{\prime}\right\|^{t} .
$$


If $t<0$, then $C^{t} \leq 1$ and so

$$
C^{t}\left\|\phi_{\omega}^{\prime}\right\|^{t}\left\|\phi_{\tau}^{\prime}\right\|^{t} \leq\left\|\phi_{\omega \tau}^{\prime}\right\|^{t} \leq C^{-t}\left\|\phi_{\omega}^{\prime}\right\|^{t}\left\|\phi_{\tau}^{\prime}\right\|^{t} .
$$

This yields the assertion.

Let us first define the auxiliary function

$$
Z_{n}(q, t)=\sum_{|\omega|=n}\left\|\phi_{\omega}^{\prime}\right\|^{t} \hat{\mu}[\omega]^{q}
$$

for $n \in \mathbb{N}$ and $q, t \in \mathbb{R}$. Now for the ergodic measure $\hat{\mu}$ and the conformal mappings $\phi_{1}, \ldots, \phi_{N}$ we can define the topological pressure $P(q, t)$ as follows:

$$
P(q, t)=\lim _{n \rightarrow \infty} \frac{1}{n} \log Z_{n}(q, t)=\lim _{n \rightarrow \infty} \frac{1}{n} \log \sum_{|\omega|=n}\left\|\phi_{\omega}^{\prime}\right\|^{t} \hat{\mu}[\omega]^{q}
$$

for $q, t \in \mathbb{R}$. The limit above exists by the standard theory of subadditive sequences since, using Lemma 2.3 and the bounded distortion property of the ergodic measure $\hat{\mu}$, we have

$$
\begin{array}{r}
C^{-(t)} K^{-(q)} \sum_{|\omega|=n}\left\|\phi_{\omega}^{\prime}\right\|^{t} \hat{\mu}[\omega]^{q} \sum_{|\tau|=p}\left\|\phi_{\tau}^{\prime}\right\|^{t} \hat{\mu}[\tau]^{q} \leq \sum_{|\omega \tau|=n+p}\left\|\phi_{\omega \tau}^{\prime}\right\|^{t} \hat{\mu}[\omega \tau]^{q} \\
\leq C^{(t)} K^{(q)} \sum_{|\omega|=n}\left\|\phi_{\omega}^{\prime}\right\|^{t} \hat{\mu}[\omega]^{q} \sum_{|\tau|=p}\left\|\phi_{\tau}^{\prime}\right\|^{t} \hat{\mu}[\tau]^{q}
\end{array}
$$

i.e.,

$$
C^{-(t)} K^{-(q)} Z_{n}(q, t) Z_{p}(q, t) \leq Z_{n+p}(q, t) \leq C^{(t)} K^{(q)} Z_{n}(q, t) Z_{p}(q, t),
$$

where

$$
K^{(q)}= \begin{cases}K^{q} & \text { if } q \geq 0 \\ K^{-q} & \text { if } q<0\end{cases}
$$

and $K^{-(q)}=\left(K^{(q)}\right)^{-1}$, and then $K^{(q)} \geq 1$ for any $q \in \mathbb{R}$. The following proposition states the well-known properties of the function $P(q, t)$ (cf. $[\mathrm{F} 2, \mathrm{P}])$.

PROPOSITION 2.4.

(i) $P(q, t): \mathbb{R} \times \mathbb{R} \rightarrow \mathbb{R}$ is continuous.

(ii) $P(q, t)$ is strictly decreasing in each variable separately.

(iii) For fixed $q$ we have

$$
\lim _{t \rightarrow+\infty} P(q, t)=-\infty \quad \text { and } \quad \lim _{t \rightarrow-\infty} P(q, t)=+\infty .
$$

(iv) $P(q, t)$ is convex: if $q_{1}, q_{2}, t_{1}, t_{2} \in \mathbb{R}, a_{1}, a_{2} \geq 0, a_{1}+a_{2}=1$, then

$$
P\left(a_{1} q_{1}+a_{2} q_{2}, a_{1} t_{1}+a_{2} t_{2}\right) \leq a_{1} P\left(q_{1}, t_{1}\right)+a_{2} P\left(q_{2}, t_{2}\right) .
$$

Now for fixed $q, P(q, t)$ is a continuous function of $t$. Its value ranges from $-\infty$ (when $t \rightarrow+\infty$ ) to $+\infty$ (when $t \rightarrow-\infty$ ). Therefore, by the 
intermediate value theorem there is a real number $\beta$ such that $P(q, \beta)=0$. The solution $\beta$ is unique, since $P(q, \cdot)$ is strictly decreasing. This defines $\beta$ implicitly as a function of $q$ : for each $q$ there is a unique $\beta=\beta(q)$ such that $P(q, \beta(q))=0$.

The following proposition gives the well-known properties of the function $\beta(q)$ (cf. [F2, $\mathrm{P}]$ ).

Proposition 2.5. Let $\beta=\beta(q)$ be defined by $P(q, \beta(q))=0$.

(i) $\beta$ is a continuous function of the real variable $q$.

(ii) $\beta$ is strictly decreasing: if $q_{1}<q_{2}$, then $\beta\left(q_{1}\right)>\beta\left(q_{2}\right)$.

(iii) $\lim _{q \rightarrow-\infty} \beta(q)=+\infty$ and $\lim _{q \rightarrow+\infty} \beta(q)=-\infty$.

(iv) $\beta$ is convex: if $q_{1}, q_{2}, a_{1}, a_{2} \in \mathbb{R}$ with $a_{1}, a_{2} \geq 0$ and $a_{1}+a_{2}=1$, then

$$
\beta\left(a_{1} q_{1}+a_{2} q_{2}\right) \leq a_{1} \beta\left(q_{1}\right)+a_{2} \beta\left(q_{2}\right) .
$$

The function $\beta(q)$ is sometimes denoted by $T(q)$ and called the temperature function. A more general discussion of this function can be found in H-P., where our $\beta(q)$ function corresponds to $-\tau(q)$ in their notation.

For any $u=u_{1} \cdots u_{k} \in \Sigma_{*}$ we denote $J_{u}=\phi_{u}(J)$, which is called $a$ cylinder set in $J$ of length $k \geq 0$. By $\mathcal{D}_{k}$ we denote the collection of all cylinder sets in $J$ of length $k$. Let $\mathcal{D}=\bigcup_{k \geq 0} \mathcal{D}_{k}$. Clearly the Borel $\sigma$-algebra on $J$ is generated by $\mathcal{D}$. Let $\mu=\hat{\mu} \circ \pi^{-1}$. Then $\mu$ is called the image measure of $\hat{\mu}$ under $\pi$ on the self-conformal set $J$ such that for any Borel $E \subset J$,

$$
\mu(E)=\inf \left\{\sum_{i} \mu\left(U_{i}\right): E \subseteq \bigcup_{i} U_{i}, U_{i} \in \mathcal{D}\right\} .
$$

For this measure $\mu$ we will determine the quantization dimension and its relationship to the temperature function arising in the thermodynamic formalism of multifractal analysis.

3. Main result. The relationship between the quantization dimension function $D_{r}$ and the temperature function $\beta(q)$ for the probability measure $\mu$ is given by the following theorem.

THEOREM 3.1. Let $\mu$ be the image measure on the self-conformal set $J$ of the shift-invariant ergodic measure $\hat{\mu}$ on the coding space under the coding map. Let $\beta=\beta(q)$ be the temperature function of the thermodynamic formalism. For each $r \in(0,+\infty)$ choose $q_{r}$ such that $\beta\left(q_{r}\right)=r q_{r}$. Then the quantization dimension for the probability measure $\mu$ is given by

$$
D_{r}=\frac{\beta\left(q_{r}\right)}{1-q_{r}} .
$$


Lemma 3.2. Let $0<r<+\infty$ be fixed. Then there exists exactly one number $\kappa_{r} \in(0,+\infty)$ such that

$$
\lim _{n \rightarrow \infty} \frac{1}{n} \log \sum_{|\omega|=n}\left(\left\|\phi_{\omega}^{\prime}\right\|^{r} \hat{\mu}[\omega]\right)^{\kappa_{r} /\left(r+\kappa_{r}\right)}=0 .
$$

Proof. From (2) we have,

$$
P(t, r t)=\lim _{n \rightarrow \infty} \frac{1}{n} \log \sum_{|\omega|=n}\left(\left\|\phi_{\omega}^{\prime}\right\|^{r} \hat{\mu}[\omega]\right)^{t} .
$$

Proposition 2.4 says that $P(t, r t)$ is continuous, convex and strictly decreasing, and hence there exists a unique $t \in \mathbb{R}$ such that $P(t, r t)=0$.

If $t=0$ then

$$
P(0,0)=\lim _{n \rightarrow \infty} \frac{1}{n} \log \sum_{|\omega|=n} 1=\lim _{n \rightarrow \infty} \frac{1}{n} \log N^{n}=\log N>0 ;
$$

and if $t=1$ then

$$
\begin{aligned}
P(1, r 1) & =\lim _{n \rightarrow \infty} \frac{1}{n} \log \sum_{|\omega|=n}\left\|\phi_{\omega}^{\prime}\right\|^{r} \hat{\mu}[\omega] \\
& \leq \lim _{n \rightarrow \infty} \frac{1}{n} \log \sum_{|\omega|=n} s^{n r} \hat{\mu}[\omega]=r \log s<0 .
\end{aligned}
$$

Therefore by the intermediate value theorem, the unique $t \in \mathbb{R}$ for which $P(t, r t)=0$ must lie between 0 and 1 . Then $\kappa_{r}=r t /(1-t)$ satisfies the conclusion of the lemma.

Lemma 3.3. Let $0<r<+\infty$ and let $\kappa_{r}$ be as in Lemma 3.2. Then for any $n \geq 1$ we have

$$
\left(C^{(r)} K\right)^{-\kappa_{r} /\left(r+\kappa_{r}\right)} \leq \sum_{|\omega|=n}\left(\left\|\phi_{\omega}^{\prime}\right\|^{r} \hat{\mu}[\omega]\right)^{\kappa_{r} /\left(r+\kappa_{r}\right)} \leq\left(C^{(r)} K\right)^{\kappa_{r} /\left(r+\kappa_{r}\right)} .
$$

Proof. For $\omega \in \Sigma_{*}$, let $s_{\omega}=\left\|\phi_{\omega}^{\prime}\right\|^{r} \hat{\mu}[\omega]$. Then for any $\omega, \tau \in \Sigma_{*}$ with $|\omega|=n,|\tau|=p(n, p \geq 1)$, by (3) we have $\left(C^{(r)} K\right)^{-1} s_{\omega} s_{\tau} \leq s_{\omega \tau} \leq$ $\left(C^{(r)} K\right) s_{\omega} s_{\tau}$. Since $C^{(r)} K \geq 1$, it is also true that $\left(C^{(r)} K\right)^{-2} s_{\omega} s_{\tau} \leq s_{\omega \tau} \leq$ $\left(C^{(r)} K\right)^{2} s_{\omega} s_{\tau}$. Hence by the standard theory of subadditive sequences, $\lim _{n \rightarrow \infty} n^{-1} \log \sum_{|\omega|=n} s_{\omega}^{t}$ exists for any $t \in \mathbb{R}$. Let us denote this limit by $h(t)$. Hence for any $t \geq 0$ we have

$$
h(t)=\lim _{p \rightarrow \infty} \frac{1}{n p} \log \sum_{|\omega|=n p} s_{\omega}^{t},
$$

and so

$$
\lim _{p \rightarrow \infty} \frac{1}{n p} \log \left(\sum_{|\omega|=n} s_{\omega}^{t}\left(C^{(r)} K\right)^{-t}\right)^{p} \leq h(t) \leq \lim _{p \rightarrow \infty} \frac{1}{n p} \log \left(\sum_{|\omega|=n} s_{\omega}^{t}\left(C^{(r)} K\right)^{t}\right)^{p},
$$


which implies

$$
\frac{1}{n} \log \sum_{|\omega|=n} s_{\omega}^{t}\left(C^{(r)} K\right)^{-t} \leq h(t) \leq \frac{1}{n} \log \sum_{|\omega|=n} s_{\omega}^{t}\left(C^{(r)} K\right)^{t}
$$

and therefore

$$
e^{n h(t)}\left(C^{(r)} K\right)^{-t} \leq \sum_{|\omega|=n} s_{\omega}^{t} \leq e^{n h(t)}\left(C^{(r)} K\right)^{t} .
$$

Now substitute $t=\kappa_{r} /\left(r+\kappa_{r}\right)$ and note that $h(t)=0$ to obtain the assertion.

We call $\Gamma \subset \Sigma_{*}$ a finite maximal antichain if $\Gamma$ is a finite set of words in $\Sigma_{*}$ such that every $\omega \in \Sigma$ is an extension of some word in $\Gamma$, but no word of $\Gamma$ is an extension of another word in $\Gamma$. Of course, this requires the index set $\{1, \ldots, N\}$ to be finite. We will make this assumption in the remainder of this paper. By $|\Gamma|$ we denote the cardinality of $\Gamma$.

Lemma 3.4. Let $\Gamma$ be a finite maximal antichain. Then

$$
\begin{gathered}
K^{-1} \sum_{\omega \in \Gamma} \hat{\mu}[\omega] \mu \circ \phi_{\omega}^{-1} \leq \mu \leq K \sum_{\omega \in \Gamma} \hat{\mu}[\omega] \mu \circ \phi_{\omega}^{-1}, \\
\sum_{\omega \in \Gamma}\left(\left\|\phi_{\omega}^{\prime}\right\|^{r} \hat{\mu}[\omega]\right)^{\kappa_{r} /\left(r+\kappa_{r}\right)} \leq\left(C^{(r)} K\right)^{2 \kappa_{r} /\left(r+\kappa_{r}\right)},
\end{gathered}
$$

where $\kappa_{r}$ is as in Lemma 3.2 .

Proof. (a) Let us first prove $\mu \leq K \sum_{\omega \in \Gamma} \hat{\mu}[\omega] \mu \circ \phi_{\omega}^{-1}$. It is enough to prove that for any $J_{\tau} \in \mathcal{D}_{k}(k \geq 1)$,

$$
\mu\left(J_{\tau}\right) \leq K \sum_{\omega \in \Gamma} \hat{\mu}[\omega] \mu \circ \phi_{\omega}^{-1}\left(J_{\tau}\right) .
$$

Since $\Gamma$ is a finite maximal antichain, for $\tau \in \Sigma_{*}$ there exists $x \in \Gamma$ such that $\tau=x y$ for some $y \in \Sigma_{*}$. Then $J_{\tau}=J_{x y}=\phi_{x y}(J)=\phi_{x}\left(\phi_{y}(J)\right)=\phi_{x}\left(J_{y}\right)$. Hence,

$$
\begin{aligned}
\sum_{\omega \in \Gamma} \hat{\mu}[\omega] \mu \circ \phi_{\omega}^{-1}\left(J_{\tau}\right) & =\sum_{\omega \in \Gamma} \hat{\mu}[\omega] \mu \circ \phi_{\omega}^{-1}\left(\phi_{x}\left(J_{y}\right)\right)=\hat{\mu}[x] \mu \circ \phi_{x}^{-1}\left(\phi_{x}\left(J_{y}\right)\right) \\
& =\hat{\mu}[x] \mu\left(J_{y}\right)=\hat{\mu}[x]\left(\hat{\mu} \circ \pi^{-1}\right)\left(J_{y}\right)=\hat{\mu}[x] \hat{\mu}[y] \\
& \geq K^{-1} \hat{\mu}[x y]=K^{-1} \hat{\mu}[\tau]=K^{-1} \mu\left(J_{\tau}\right),
\end{aligned}
$$

so that

$$
\mu\left(J_{\tau}\right) \leq K \sum_{\omega \in \Gamma} \hat{\mu}[\omega] \mu \circ \phi_{\omega}^{-1}\left(J_{\tau}\right) \quad \text { for any } J_{\tau} \in \mathcal{D}_{k}(k \geq 1) .
$$

Similarly, it can be proved that $K^{-1} \sum_{\omega \in \Gamma} \hat{\mu}[\omega] \mu \circ \phi_{\omega}^{-1}\left(J_{\tau}\right) \leq \mu\left(J_{\tau}\right)$ for any $J_{\tau} \in \mathcal{D}_{k}(k \geq 1)$, completing the proof (a). 
To prove (b), let $m=\min \{|\omega|: \omega \in \Gamma \backslash\{\emptyset\}\}$. Then for each $\omega \in \Gamma \backslash\{\emptyset\}$ there exists $\tau(\omega) \in \Sigma_{*}$ with $|\tau(\omega)|=m$ and $\tau(\omega) \prec \omega$, i.e., there exists $x(\omega) \in \Sigma_{*}$ such that $\omega=\tau(\omega) x(\omega)$. Now for any $\omega \in \Gamma$ we can write

$$
\begin{aligned}
\left\|\phi_{\omega}^{\prime}\right\| & \leq C\left\|\phi_{\tau(\omega)}^{\prime}\right\|\left\|\phi_{x(\omega)}^{\prime}\right\| \leq C\left\|\phi_{\tau(\omega)}^{\prime}\right\|, \\
\hat{\mu}[\omega] & \leq K \hat{\mu}[\tau(\omega)] \hat{\mu}\left[x_{\omega}\right] \leq K \hat{\mu}[\tau(\omega)] .
\end{aligned}
$$

From the above inequalities and Lemma 3.3 we have

$$
\begin{aligned}
\sum_{\omega \in \Gamma}\left(\left\|\phi_{\omega}^{\prime}\right\|^{r} \hat{\mu}[\omega]\right)^{\kappa_{r} /\left(r+\kappa_{r}\right)} & \leq\left(C^{(r)} K\right)^{\kappa_{r} /\left(r+\kappa_{r}\right)} \sum_{\omega \in \Gamma}\left(\left\|\phi_{\tau(\omega)}^{\prime}\right\|^{r} \hat{\mu}[\tau(\omega)]\right)^{\kappa_{r} /\left(r+\kappa_{r}\right)} \\
& \leq\left(C^{(r)} K\right)^{\kappa_{r} /\left(r+\kappa_{r}\right)} \sum_{|\tau|=m}\left(\left\|\phi_{\tau}^{\prime}\right\|^{r} \hat{\mu}[\tau]\right)^{\kappa_{r} /\left(r+\kappa_{r}\right)} \\
& \leq\left(C^{(r)} K\right)^{2 \kappa_{r} /\left(r+\kappa_{r}\right)} .
\end{aligned}
$$

Lemma 3.5. Let $\Gamma \subset \Sigma_{*}$ be a finite maximal antichain, $n \in \mathbb{N}$ with $n \geq|\Gamma|$, and $0<r<+\infty$. Then

$$
V_{n, r}(\mu) \leq \inf \left\{\tilde{C}^{r} K \sum_{\omega \in \Gamma}\left\|\phi_{\omega}^{\prime}\right\|^{r} \hat{\mu}[\omega] V_{n_{\omega}, r}(\mu): n_{\omega} \geq 1, \sum_{\omega \in \Gamma} n_{\omega} \leq n\right\} .
$$

Proof. Suppose $n_{\omega} \geq 1$ for each $\omega \in \Gamma$, and $\sum_{\omega \in \Gamma} n_{\omega} \leq n$. For each $\omega \in \Gamma$ let $\alpha_{\omega}$ be an $n_{\omega}$-optimal set for $V_{n_{\omega}, r}(\mu)$.

Since $\left|\bigcup_{\omega \in \Gamma} \phi_{\omega}\left(\alpha_{\omega}\right)\right| \leq n$ and $\mu \leq K \sum_{\omega \in \Gamma} \hat{\mu}[\omega] \mu \circ \phi_{\omega}^{-1}$, we have

$$
\begin{aligned}
V_{n, r}(\mu) & \leq \int d\left(x, \bigcup \phi_{\omega}\left(\alpha_{\omega}\right)\right)^{r} d \mu(x) \\
& \leq K \sum_{\omega \in \Gamma} \hat{\mu}[\omega] \int d\left(x, \bigcup \phi_{\omega}\left(\alpha_{\omega}\right)\right)^{r} d\left(\mu \circ \phi_{\omega}^{-1}\right)(x) \\
& \leq K \sum_{\omega \in \Gamma} \hat{\mu}[\omega] \int d\left(\phi_{\omega}(x), \phi_{\omega}\left(\alpha_{\omega}\right)\right)^{r} d \mu(x) \\
& \leq \tilde{C}^{r} K \sum_{\omega \in \Gamma}\left\|\phi_{\omega}^{\prime}\right\|^{r} \hat{\mu}[\omega] \int d\left(x, \alpha_{\omega}\right)^{r} d \mu(x) \quad \text { by Lemma 2.2 } \\
& =\tilde{C}^{r} K \sum_{\omega \in \Gamma}\left\|\phi_{\omega}^{\prime}\right\|^{r} \hat{\mu}[\omega] V_{n_{\omega}, r}(\mu),
\end{aligned}
$$

which implies the lemma.

Proposition 3.6. Let $0<r<+\infty$ and let $\kappa_{r}$ be as in Lemma 3.2. Then $\lim \sup _{n \rightarrow \infty} n e_{n, r}^{\kappa_{r}}<+\infty$.

Proof. Let $q_{r}=\kappa_{r} /\left(r+\kappa_{r}\right)$; then $\beta\left(q_{r}\right)=r q_{r}$. Choose $\epsilon_{0}$ so that $0<$ $\epsilon_{0}<1$. Fix $m \in \mathbb{N}$. Choose any $n \in \mathbb{N}$ so that $m / n<\epsilon_{0}$, and set $\epsilon=\epsilon_{0} m / n$, so that $0<\epsilon<1$. Let

$$
\Gamma=\Gamma(\epsilon)=\left\{\omega \in \Sigma_{*}:\left(\left\|\phi_{\omega}^{\prime}\right\|^{r} \hat{\mu}[\omega]\right)^{\kappa_{r} /\left(r+\kappa_{r}\right)}<\epsilon \leq\left(\left\|\phi_{\omega^{-}}^{\prime}\right\|^{r} \hat{\mu}\left[\omega^{-}\right]\right)^{\kappa_{r} /\left(r+\kappa_{r}\right)}\right\} .
$$

Since the index set $\{1, \ldots, N\}$ is finite, $\Gamma$ is a finite maximal antichain. 
Hence by the previous lemma we have

$$
\begin{aligned}
V_{n, r}(\mu) & \leq \tilde{C}^{r} K \sum_{\omega \in \Gamma}\left\|\phi_{\omega}^{\prime}\right\|^{r} \hat{\mu}[\omega] V_{m, r}(\mu) \\
& =\tilde{C}^{r} K \sum_{\omega \in \Gamma}\left(\left\|\phi_{\omega}^{\prime}\right\|^{r} \hat{\mu}[\omega]\right)^{\kappa_{r} /\left(r+\kappa_{r}\right)}\left(\left\|\phi_{\omega}^{\prime}\right\|^{r} \hat{\mu}[\omega]\right)^{r /\left(r+\kappa_{r}\right)} V_{m, r}(\mu) \\
& <\tilde{C}^{r} K \sum_{\omega \in \Gamma}\left(\left\|\phi_{\omega}^{\prime}\right\|^{r} \hat{\mu}[\omega]\right)^{\kappa_{r} /\left(r+\kappa_{r}\right)} \epsilon^{r / \kappa_{r}} V_{m, r}(\mu) \\
& \leq \tilde{C}^{r} K\left(C^{(r)} K\right)^{2 \kappa_{r} /\left(r+\kappa_{r}\right)} \epsilon^{r / \kappa_{r}} V_{m, r}(\mu) \quad \text { (by Lemma 3.4 } \\
& =\tilde{C}^{r} K\left(C^{(r)} K\right)^{2 \kappa_{r} /\left(r+\kappa_{r}\right)} \epsilon_{0}^{r / \kappa_{r}}(m / n)^{r / \kappa_{r}} V_{m, r}(\mu),
\end{aligned}
$$

and therefore

$$
n V_{n, r}^{\kappa_{r} / r}(\mu) \leq\left(\tilde{C}^{r} K\right)^{\kappa_{r} / r}\left(C^{(r)} K\right)^{2 \kappa_{r}^{2} /\left(r\left(r+\kappa_{r}\right)\right)} \epsilon_{0} m V_{m, r}^{\kappa_{r} / r}(\mu) .
$$

Since the inequality holds for all but a finite number of $n$, we have

$$
\limsup _{n \rightarrow \infty} n e_{n, r}^{\kappa_{r}} \leq\left(\tilde{C}^{r} K\right)^{\kappa_{r} / r}\left(C^{(r)} K\right)^{2 \kappa_{r}^{2} /\left(r\left(r+\kappa_{r}\right)\right)} \epsilon_{0} m e_{m, r}^{\kappa_{r}}<+\infty .
$$

Lemma 3.7. Let $\Gamma \subset \Sigma_{*}$ be a finite maximal antichain. Then there exists $n_{0}=n_{0}(\Gamma)$ such that for every $n \geq n_{0}$ there exists a set $\left\{n_{\omega}:=n_{\omega}(n)\right\}_{\omega \in \Gamma}$ of positive integers such that $\sum_{\omega \in \Gamma} n_{\omega} \leq n$ and

$$
u_{n, r} \geq\left(\tilde{C}^{r} K\right)^{-1} \sum_{\omega \in \Gamma}\left\|\phi_{\omega}^{\prime}\right\|^{r} \hat{\mu}[\omega] u_{n_{\omega}, r} .
$$

Proof. Let $U$ be the open set from the strong open set condition. Then there exists $\tau \in \Sigma_{*}$ such that $\phi_{\tau}(X) \subset U$. Let $\epsilon=d\left(\phi_{\tau}(X), U^{c}\right)$ and $\lambda=\tilde{C}^{-1} \min _{\omega \in \Gamma}\left\{\left\|\phi_{\omega}^{\prime}\right\|\right\}$. Then for $\omega \in \Gamma$ we have $d\left(\phi_{\omega} \phi_{\tau}(X), \phi_{\omega}\left(U^{c}\right)\right) \geq$ $\tilde{C}^{-1}\left\|\phi_{\omega}^{\prime}\right\| d\left(\phi_{\tau}(X), U^{c}\right) \geq \lambda \epsilon$, which implies $d\left(x, U^{c}\right) \geq d\left(x, \phi_{\omega}\left(U^{c}\right)\right) \geq \lambda \epsilon$ for any $x \in \phi_{\omega}\left(\phi_{\tau}(X)\right)$. For each $n$, let $\alpha_{n}$ be an $n$-optimal set for $u_{n, r}$ and let $\delta_{n}=\max \left\{d\left(x, \alpha_{n} \cup U^{c}\right): x \in J\right\}$. Since $\delta_{n} \rightarrow 0$ as $n \rightarrow \infty$ we can choose $n_{0}$ such that $\delta_{n}<\lambda \epsilon$ for all $n \geq n_{0}$. Suppose $n \geq n_{0}$ and $x \in \phi_{\omega}\left(\phi_{\tau}(J)\right)$. There exists $a \in \alpha_{n} \cup U^{c}$ such that $d\left(x, \alpha_{n} \cup U^{c}\right)=d(x, a) \leq \delta_{n}<\lambda \epsilon$, and so $a \in \phi_{\omega}(U)$. Therefore, letting $\alpha_{n_{\omega}}=\alpha_{n} \cap \phi_{\omega}(U)$, we get $n_{\omega}:=\left|\alpha_{n_{\omega}}\right| \geq 1$ and $\sum_{\omega \in \Gamma} n_{\omega} \leq n$. Hence,

$$
\begin{aligned}
u_{n, r} & =\int d\left(x, \alpha_{n} \cup U^{c}\right)^{r} d \mu(x) \geq K^{-1} \sum_{\omega \in \Gamma} \hat{\mu}[\omega] \int d\left(\phi_{\omega}(x), \alpha_{n} \cup U^{c}\right)^{r} d \mu(x) \\
& \geq K^{-1} \sum_{\omega \in \Gamma} \hat{\mu}[\omega] \int d\left(\phi_{\omega}(x), \alpha_{n} \cup \phi_{\omega}\left(U^{c}\right)\right)^{r} d \mu(x) \\
& =K^{-1} \sum_{\omega \in \Gamma} \hat{\mu}[\omega] \int d\left(\phi_{\omega}(x), \alpha_{n_{\omega}} \cup \phi_{\omega}\left(U^{c}\right)\right)^{r} d \mu(x)
\end{aligned}
$$




$$
\begin{aligned}
& \geq K^{-1} \tilde{C}^{-r} \sum_{\omega \in \Gamma}\left\|\phi_{\omega}^{\prime}\right\|^{r} \hat{\mu}[\omega] \int d\left(x, \phi_{\omega}^{-1}\left(\alpha_{n_{\omega}}\right) \cup U^{c}\right)^{r} d \mu(x) \\
& \geq\left(\tilde{C}^{r} K\right)^{-1} \sum_{\omega \in \Gamma}\left\|\phi_{\omega}^{\prime}\right\|^{r} \hat{\mu}[\omega] u_{n_{\omega}, r} .
\end{aligned}
$$

Proposition 3.8. Let $\left\{\phi_{1}, \ldots, \phi_{N}\right\}$ satisfy the strong open set condition and let $0<r<+\infty$. Moreover, let $\kappa_{r}$ be as in Lemma 3.2. Let $0<\ell<\kappa_{r}$. Then $\liminf _{n \rightarrow \infty} n e_{n, r}^{\ell}>0$.

Proof. Since $0<\ell<\kappa_{r}$ and $\kappa_{r}$ is unique for which

$$
\lim _{n \rightarrow \infty} \frac{1}{n} \log \sum_{|\omega|=n}\left(\left\|\phi_{\omega}^{\prime}\right\|^{r} \hat{\mu}[\omega]\right)^{\kappa_{r} /\left(r+\kappa_{r}\right)}=0,
$$

we have

$$
\sum_{|\omega|=m}\left(\left\|\phi_{\omega}^{\prime}\right\|^{r} \hat{\mu}[\omega]\right)^{\ell /(r+\ell)} \rightarrow \infty \quad \text { as } m \rightarrow \infty .
$$

Choose $m$ so that the above sum is greater than 1 and let $\Gamma=\left\{\omega \in \Sigma_{*}\right.$ : $|\omega|=m\}$. Then $\Gamma$ is a finite maximal antichain. The previous lemma yields an $n_{0}$ and for $n \geq n_{0}$ the numbers $\left\{n_{\omega}:=n_{\omega}(n)\right\}_{\omega \in \Gamma}$ which satisfy the conclusion of that lemma. Set $c=\min \left\{n^{r / \ell} u_{n, r}: n \leq n_{0}\right\}$. Clearly each $u_{n, r}>0$ and hence $c>0$. Suppose $n \geq n_{0}$ and $k^{r / \ell} u_{k, r} \geq c$ for all $k<n$. Using the previous lemma we have

$$
\begin{aligned}
n^{r / \ell} u_{n, r} & \geq n^{r / \ell}\left(\tilde{C}^{r} K\right)^{-1} \sum_{\omega \in \Gamma}\left\|\phi_{\omega}^{\prime}\right\|^{r} \hat{\mu}[\omega] u_{n_{\omega}, r} \\
& =n^{r / \ell}\left(\tilde{C}^{r} K\right)^{-1} \sum_{\omega \in \Gamma}\left\|\phi_{\omega}^{\prime}\right\|^{r} \hat{\mu}[\omega]\left(n_{\omega}(n)\right)^{-r / \ell}\left(n_{\omega}(n)\right)^{r / \ell} u_{n_{\omega}, r} \\
& \geq c\left(\tilde{C}^{r} K\right)^{-1} \sum_{\omega \in \Gamma}\left\|\phi_{\omega}^{\prime}\right\|^{r} \hat{\mu}[\omega]\left(\frac{n_{\omega}(n)}{n}\right)^{-r / \ell} .
\end{aligned}
$$

Using Hölder's inequality (with exponents less than 1) we have

$$
\begin{aligned}
& n^{r / \ell} u_{n, r} \\
& \quad \geq c\left(\tilde{C}^{r} K\right)^{-1}\left(\sum_{\omega \in \Gamma}\left(\left\|\phi_{\omega}^{\prime}\right\|^{r} \hat{\mu}[\omega]\right)^{\ell /(r+\ell)}\right)^{1+r / \ell}\left(\sum_{\omega \in \Gamma}\left(\frac{n_{\omega}(n)}{n}\right)^{(-r / \ell)(-\ell / r)}\right)^{-r / \ell} .
\end{aligned}
$$

By our choice of $\Gamma$, which depends only on $\ell$ and not on $n$, and the fact that $\sum_{\omega \in \Gamma} n_{\omega}(n) \leq n$, we see that $n^{r / \ell} u_{n, r} \geq c\left(\tilde{C}^{r} K\right)^{-1}$. Hence, by induction,

$$
\liminf _{n \rightarrow \infty} n u_{n, r}^{\ell / r} \geq\left(c\left(\tilde{C}^{r} K\right)^{-1}\right)^{\ell / r}>0, \quad \text { i.e. } \quad \liminf _{n \rightarrow \infty} n e_{n, r}^{\ell}>0 .
$$

Proof of Theorem 3.1. From Proposition 11.3 of [GL1] we know that:

(a) If $0 \leq t<\underline{D}_{r}<s$ then

$$
\lim _{n \rightarrow \infty} n e_{n, r}^{t}=+\infty \quad \text { and } \quad \liminf _{n \rightarrow \infty} n e_{n, r}^{s}=0 .
$$


(b) If $0 \leq t<\bar{D}_{r}<s$ then

$$
\limsup _{n \rightarrow \infty} n e_{n, r}^{t}=+\infty \quad \text { and } \quad \lim _{n \rightarrow \infty} n e_{n, r}^{s}=0 .
$$

From (a) and Proposition 3.8 we have $\ell \leq \underline{D}_{r}$ whenever $\ell<\kappa_{r}$. Hence $\kappa_{r} \leq \underline{D}_{r}$. From (b) and Proposition 3.6 we have $\bar{D}_{r} \leq \kappa_{r}$. Hence $\kappa_{r} \leq \underline{D}_{r} \leq$ $\bar{D}_{r} \leq \kappa_{r}$, i.e., the quantization dimension $D_{r}$ exists and $D_{r}=\kappa_{r}$. Note that for $q_{r}=\kappa_{r} /\left(r+\kappa_{r}\right)$ and $\beta\left(q_{r}\right)=r q_{r}$ we have $D_{r}=\beta\left(q_{r}\right) /\left(1-q_{r}\right)$. This completes the proof of the theorem.

Acknowledgments. The author would like to express his sincere thanks to Professor R. Daniel Mauldin of the University of North Texas for introducing him to quantization theory and iterated function systems.

\section{References}

[F1] K. J. Falconer, Techniques in Fractal Geometry, Wiley, Chichester, 1997.

[F2] - The multifractal spectrum of statistically self-similar measures, J. Theoret. Probab. 7 (1994), 681-701.

[GG] A. Gersho and R. M. Gray, Vector Quantization and Signal Compression, Kluwer, 1992.

[GL1] S. Graf and H. Luschgy, Foundations of Quantization for Probability Distributions, Lecture Notes in Math. 1730, Springer, Berlin, 2000.

[GL2] - - - Asymptotics of the quantization errors for self-similar probabilities, Real Anal. Exchange 26 (2001), 795-810.

[H-P] T. Halsey, M. Jensen, L. Kadanoff and I. Procaccia, Fractal measures and their singularities: the characterization of strange sets, Phys. Rev. A 33 (1986), 11411151; Erratum, ibid. 34 (1986), 1601.

[LM] L. J. Lindsay and R. D. Mauldin, Quantization dimension for conformal iterated function systems, Nonlinearity 15 (2002), 189-199.

[P] N. Patzschke, Self-conformal multifractal measures, Adv. Appl. Math. 19 (1997), 486-513.

[P-S] Y. Peres, M. Rams, K. Simon and B. Solomyak, Equivalence of positive Hausdorff measure and the open set condition for self-conformal sets, Proc. Amer. Math. Soc. 129 (2001), 2689-2699.

[R] M. K. Roychowdhury, Quantization dimension function and Gibbs measure, preprint.

Mrinal Kanti Roychowdhury

Department of Mathematics

The University of Texas-Pan American

1201 West University Drive

Edinburg, TX 78539-2999, U.S.A.

E-mail: roychowdhurymk@utpa.edu 